ISSN-L 2077-0014

DOI: https://doi.org/10.33326/26176068.2021.3.1158

Artículo original/Original article/Artigo original

\title{
Relación entre anemia ferropénica y metales pesados o parásitos en adolescentes que consumen agua subterránea
}

Relationship between iron-deficiency anemia and heavy metals or parasites in adolescents who consume groundwater

Relação entre anemia ferropénica e metais pesados ou parasitas em adolescentes que consomem água subterrânea

Daisy Rossy Bustinza-Salas ${ }^{1}$

(D) https://orcid.org/0000-0002-7274-5437

\begin{abstract}
Resumen
Objetivo: Determinar la relación entre anemia ferropénica y la presencia de metales pesados o parásitos en adolescentes que consumen agua subterránea en la ciudad de Juliaca, Puno. Material y métodos: Estudio prospectivo y analítico. Se analizó la concentración de arsénico y aluminio en agua subterránea por espectrofotometría por emisión óptica en 24 muestras, la concentración de arsénico y aluminio en orina por espectrofotometría de masas acoplado en plasma inductivo en 24 muestras; presencia o ausencia de Giardia lamblia y Entamoeba histolytica en análisis parasitológicos directos en 24 muestras de agua subterránea; exámenes hematológicos de hemoglobina, hematocrito y constantes corpusculares en 92 adolescentes residentes de la Urbanización Taparachi, durante el año 2019. Resultados: Se encontró niveles permisibles de arsénico en agua subterránea, se encontró niveles de hemoglobina, hematocrito y constantes corpusculares dentro de los valores de referencia considerados como normales en los adolescentes. Sin embargo, se encontró niveles de arsénico por encima de los valores aceptables en orina de los adolescentes, estos valores encontrados son por un tipo de arsénico orgánico o los adolescentes están siendo tolerantes a niveles muy bajos de arsénico inorgánico siendo permisibles en agua subterránea. Se encontraron niveles aceptables de aluminio en orina en los adolescentes, mientras que los valores no permisibles de aluminio en agua subterránea son por cualquier otra causa externa. Se demostró que no existe presencia de Giardia lamblia, Entamoeba histolytica en agua subterránea. Conclusión: No existe relación entre anemia ferropénica y consumo de agua subterránea en adolescentes.
\end{abstract}

Palabras clave: aluminio, arsénico, anemia ferropénica, adolescente

\begin{abstract}
Objective: To determine the relationship between iron deficiency anemia and the presence of heavy metals or parasites in adolescents who consume groundwater in Juliaca, Puno. Material and methods: Prospective and analytical study. The concentration of arsenic and aluminium in groundwater was analyzed by spectrophotometry by optical emission in 24 samples, the concentration of arsenic and aluminium in urine by mass spectrophotometry coupled in inductive plasma in 24 samples; presence or absence of Giardia lamblia and Entamoeba histolytica in direct parasitological analyses in 24 groundwater samples; haematoglobin, hematocrit and corpuscular constants tests in 92 adolescent residents of the Taparachi urbanization, during the year 2019. Results: Permissible levels of arsenic were found in groundwater, hemoglobin, hematocrit and corpuscular constants were found within the reference values considered normal in adolescents. However, arsenic levels were found to be above acceptable levels in adolescent urine, these values are found by a type of organic arsenic or adolescents are being tolerant to very low levels of inorganic arsenic being permissible in groundwater. Acceptable levels of aluminum in urine were found in adolescents, while the impermissible values of aluminum in groundwater are for any other external cause. It was shown that there is no presence of Giardia lamblia and Entamoeba histolytica in groundwater. Conclusion: There is no relationship between iron deficiency anemia and groundwater consumption in adolescents.
\end{abstract}

Keywords: aluminum, arsenic, iron deficiency anemia, adolescent

${ }^{1}$ Universidad Andina Néstor Cáceres Velásquez. Tecnóloga médica. Juliaca, Perú 


\begin{abstract}
Resumo
Objetivo: Determinar a relação entre anemia ferropénica e a presença de metais pesados ou parasitas em adolescentes que consomem água subterrânea na cidade de Juliaca, Puno. Material e métodos: Estudo prospectivo e analítico. A concentração de arsénio e alumínio nas águas subterrâneas foi analisada por espectrofotometria de emissão óptica em 24 amostras, a concentração de arsénio e alumínio na urina por espectrofotometria de massa acoplada a plasma indutivo em 24 amostras; presença ou ausência de Giardia lamblia e Entamoeba histolytica em análises parasitárias diretas em 24 amostras de água subterrânea; exames hematológicos de hemoglobina, hematócrito e constantes corpusculares em 92 adolescentes residentes da Urbanização Taparachi, durante o ano de 2019. Resultados: Foram encontrados níveis admissíveis de arsênico nas águas subterrâneas, níveis de hemoglobina, hematócrito e constantes corpusculares foram encontrados dentro dos valores de referência considerados normais em adolescentes. No entanto, foram encontrados níveis de arsênico acima dos valores aceitáveis na urina dos adolescentes, estes valores encontrados são por um tipo de arsênico orgânico ou os adolescentes estão sendo tolerantes a níveis muito baixos de arsênico inorgânico sendo permissíveis em águas subterrâneas. Foram encontrados níveis aceitáveis de alumínio na urina em adolescentes, enquanto os valores não admissíveis de alumínio em água subterrânea são por qualquer outra causa externa. Foi demonstrado que não há presença de Giardia lamblia e Entamoeba histolytica em águas subterrâneas. Conclusão: Não existe relação entre anemia ferropénica e consumo de água subterrânea em adolescentes.
\end{abstract}

Palavras-chave: alumínio, arsênico, anemia ferropénica, adolescente

\section{Introducción}

El consumo de agua subterránea se da con incidencia en todo el mundo siendo un medio para obtener agua. La anemia ferropénica es de causa en la deficiencia de hierro. Su importancia no solo radica en la deficiencia de hierro sino también en los factores que la ocasionan, aún en su modo leve. No es un problema actual, pudiendo afectar a más consumidores de este tipo de agua.

Las causas de la anemia ferropénica pueden ser por la falta de hierro en el organismo, producto tanto de deficiencia nutricional o absortiva, como de pérdida crónica de sangre causa anemia ferropénica, siempre progresivamente microcítica e hipocrómica. Por otro lado, está la falta de oferta de hierro a la eritropoyesis, por retención preferencial en las células reticulares y macrofágicas. Esa ferropenia funcional es parte de la patogénesis múltiple de la anemia de las enfermedades crónicas, inicialmente normocítica, después microcítica. De otro modo, los adolescentes al encontrarse en desarrollo y con niveles bajos de hemoglobina pueden tener deficiencias en el rendimiento de sus actividades diarias. ${ }^{2}$

El arsénico (As) de número atómico 33 y peso atómico 74,922 es un elemento ampliamente distribuido en la atmósfera, en la hidrosfera y en la biosfera. Si bien una gran cantidad de As en el medio ambiente provienedefuentesnaturales (meteorización, actividad biológica, emisiones volcánicas), existe una importante contribución a partir de actividades antropogénicas, tales como procesos industriales como la minería y la fundición de metales, o el uso en biocidas y en conservantes de la madera. La toxicidad depende de la especie química, incluidas las especies orgánicas. En el agua, los niveles de As son más elevados en aguas subterráneas, especialmente en áreas con depósitos de roca volcánica o de minerales ricos en As. La hemoglobina baja, moderada y severa es un principal indicador de exposición al arsénico. La ruta de exposición al arsénico se da principalmente por el consumo, dándose la acumulación de arsénico en hígado, riñones y su excreción por la orina. En dosis con altas concentraciones de arsénico se acumulan en tejidos adiposos, cabello, uñas entre otros órganos. ${ }^{3}$

El aluminio (Al) se encuentra ampliamente distribuido en la naturaleza. Una proporción natural de $8 \%$ de la superficie terrestre lo ubica en el primer lugar de abundancia relativa entre los metales y el tercero entre todos los elementos de la corteza. La combinación de su disponibilidad con propiedades mecánicas y eléctricas únicas, aseguran a la química un futuro brillante y en constante expansión. Afirman haber observado una asociación entre alteraciones de la integridad de proteínas de la membrana eritrocitaria y la aparición de anomalías morfológicas, asimismo, demuestran que el Al, el cual comparte con el hierro la proteína de transporte transferrina, interfiere con los mecanismos celulares de captación de hierro y con la síntesis de hemoglobina. ${ }^{4}$

Las pérdidas anormales de hierro se pueden dar como consecuencia de la infestación por Entamoeba histolytica, parásito que provoca hemorragia crónica en el intestino. Además, puede haber una mala absorción del hierro debido a la presencia de Giardia 
lamblia. Son tres las vías reconocidas, siguiendo la ruta fecal oral: a través del agua, los alimentos y por contacto de persona a persona. Las materias fecales humanas y/o de animales son las fuentes de contaminación. ${ }^{5}$ El principal mecanismo de acción patógena, en giardiasis, se debe a la acción de los parásitos sobre la mucosa del intestino delgado, principalmente del duodeno y yeyuno. Esta acción se hace por fijación de los trofozoítos por medio de la ventosa y da origen a inflamación catarral. La patología principal se encuentra en infecciones masivas, en cuyo caso la barrera mecánica creada por los parásitos y la inflamación intestinal, pueden llegar a producir un síndrome de malabsorción. En estos casos las vellosidades intestinales se encuentran atrofiadas, hay inflamación de la lámina propia, y alteraciones morfológicas de las células epiteliales. El trofozoíto de Entamoeba histolytica se encuentra en la luz del colon o invadiendo la pared intestinal, donde se reproduce por división binaria simple. ${ }^{6}$

El objetivo de este estudio es determinar la relación entre anemia ferropénica y la presencia de metales pesados o parásitos en adolescentes que consumen agua subterránea en la ciudad de Juliaca, Puno.

\section{Material y métodos}

Estudio prospectivo y analítico. Se seleccionó 92 adolescentes y por 24 muestras de orina y de agua subterránea de la urbanización Taparachi de la ciudad de Juliaca en Puno, de forma voluntaria, durante el año 2019. Se incluyó a adolescentes previo consentimiento informado de sus padres, que consumieron agua subterránea sin ningún químico añadido antes, que vivan de forma permanente en la Urbanización Taparachi III, mujeres que no se encuentren en periodo menstrual y no presenten riesgo de embarazo y que no hayan consumido en las últimas dos semanas alimentos ricos en arsénico orgánico. Se excluyó a los adolescentes que hayan tenido alguna cirugía en los últimos tres meses o se encuentren en tratamiento.

Las variables de estudio fueron: consumo de agua subterránea en adolescentes de la urbanización Taparachi III, siendo los indicadores de medición la edad, peso, talla, género, arsénico, aluminio y Giardia lamblia y Entamoeba histolytica de los adolescentes; y la variable anemia ferropénica, con sus indicadores hemoglobina, hematocrito, volumen corpuscular media, hemoglobina corpuscular media y concentración de la hemoglobina corpuscular media.
Los instrumentos de evaluación que se utilizaron fueron: espectrofotometría de masas con plasma acoplado por inducción, espectrofotometría de emisión óptica, hemograma automatizado por citometría de flujo y prueba parasitológico directo.

Para medir la concentración del arsénico y aluminio en agua subterránea se realizó pruebas en el laboratorio, con esta finalidad se utilizaron las pruebas bioquímicas basadas en el índice de absorbancia por espectrofotometría de emisión óptica. Según la Organización Mundial de la Salud (OMS), los valores referenciales son 0,01 $\mathrm{mg} / \mathrm{L}$ para arsénico en agua subterránea, mientras que, los valores referenciales para aluminio en agua subterránea son $0,2 \mathrm{mg} / \mathrm{L}^{7}$

La recolección de las muestras se hizo en la Urbanización Taparachi III, se ubicó a 24 adolescentes que participarían voluntariamente en este estudio, luego se hizo la recolección de las muestras, así se tomó de cada pozo 5 litros de agua subterránea en unas botellas anteriormente esterilizadas. Posteriormente fueron llevadas 24 muestras de agua subterránea al laboratorio. Las muestras fueron procesadas por el equipo automatizado de espectrofotometría de emisión óptica:

1. Se pasó un control de calidad de equipo y reactivos.

2. Las muestras de agua fueron pasadas por cubetas en el canal del equipo, posteriormente el equipo midió la concentración de arsénico y aluminio. Se anotó los resultados y a que adolescente le pertenece.

Para medir la concentración del arsénico y el aluminio en orina se realizó pruebas en el laboratorio, así se utilizó la espectrofotometría de masas con plasma acoplado por inducción, que midió la concentración del aluminio y el arsénico en la orina simple. Según el índice de exposición biológica, los intervalos de referencia son referidos como $\mathrm{BEI}$, para arsénico y aluminio en orina los valores de referencia son menor o igual a $50 \mathrm{ug} / \mathrm{gramo}$ de creatinina. ${ }^{8}$

Los adolescentes de quienes se midió su concentración del agua subterránea que consumen, participaron dando su muestra de orina simple. Para la recolección de orina simple se les indico que tres días antes no consuman mariscos, bacalaos ni pescados, pues estos alimentos son muy ricos en arsénico orgánico. Aunque el arsénico orgánico es de muy baja toxicidad, esta metodología también la mide. Posterior a eso se tomó 24 muestras de orina simple de la mañana en frascos herméticos estériles 
de los adolescentes que oscilan entre 13 a 17 años de edad. Estás muestras fueron llevadas al laboratorio inmediatamente en cooler. En el laboratorio se realizó:

1. Se pasó por un control de calidad para la utilización de los reactivos y equipo.

2. Después se pasó las muestras de orinas simples en cubetas por el equipo automatizado de espectrofotometría de masas con plasma acoplado por inducción. El equipo emitió la concentración de cada muestra, se anotó, evaluó y comparó cada resultado.

Para medir la hemoglobina, hematocrito y constantes corpusculares se realizó el hemograma automatizado por citometría de flujo. En ese sentido, se realizó la toma de muestra a los adolescentes en la urbanización Taparachi III.

1. Se hizo la identificación del adolescente, teniendo variables como nombre, edad, género y la firma del consentimiento informado. Posterior a eso se tomó 92 muestras de sangre venosa con agujas estériles, en unos tubos que contienen EDTA. Luego las muestras fueron llevados al laboratorio.

2. En el laboratorio se pasó los controles de calidad para reactivos y equipo.

3. Después los tubos de EDTA pasaron por el canal del equipo.

4. El equipo midió y emitió la concentración de hemoglobina, hematocrito, constantes corpusculares. Se anotó, evaluó y comparó.

La altura de la ciudad, Juliaca se encuentra a 3800 metros sobre el nivel del mar; considerando así los valores de referencia para hemoglobina en mujeres desde $14 \mathrm{mg} / \mathrm{dl} \mathrm{a} 17 \mathrm{mg} / \mathrm{dl}$, y en adolescentes varones los valores de referencia son a partir de 15 $\mathrm{mg} / \mathrm{dl}$ hasta $18 \mathrm{mg} / \mathrm{dl}$. Mientras que, los valores a partir de $13 \mathrm{mg} / \mathrm{dl}$ en adelante serán considerados como leve, los valores a partir de $11 \mathrm{mg} / \mathrm{dl}$ en adelante serán considerados como moderado, los valores a partir de $10 \mathrm{mg} / \mathrm{dl}$ para abajo serán considerados como severos, y los valores a partir de $18 \mathrm{mg} / \mathrm{dl}$ en adelante serán considerados como ligeramente elevados.

Para hematocrito los valores de referencia para adolescentes mujeres son desde 44 a $54 \%$, valores menores a estos son considerados como bajos, mientras que, en adolescentes varones los valores de referencia son a partir de $45 \%$ hasta 55 $\%$. Valores menores a estos son considerados como bajos.

Los valores de referencia para volumen corpuscular medio en adolescentes son desde $80 \mathrm{fl}$ hasta $96 \mathrm{fl}$. Valores inferiores a estos son considerados como bajos.

Para hemoglobina corpuscular media los valores de referencia para adolescentes serán a partir desde $33 \mathrm{pg}$ hasta $36 \mathrm{pg}$. Valores inferiores a estos serán considerados como bajos.

Para medir la concentración corpuscular media de hemoglobina consideraremos los valores de referencia para adolescentes a partir desde 33 $\mathrm{g} / \mathrm{dL}$ hasta $36 \mathrm{~g} / \mathrm{dL}$. Valores inferiores a estos son considerados como bajos.

Para hallar la presencia o ausencia de la Giardia lamblia y Entamoeba histolytica se realizó pruebas de laboratorio, con esta finalidad se utilizó las pruebas microbiológicas basadas en el índice conocido como parasitológico de forma directa que relaciona la presencia según estructura de Giardia lamblia y Entamoeba histolytica o no en el agua subterránea.

Para el análisis parasitológico directo se recolectó 24 muestras de agua subterránea:

1. Se realizó la sedimentación del agua subterránea, así se dejó tres días en botellas anteriormente esterilizadas. Al cuarto día se decantó y se trabajó con el sedimento.

2. Se colocó el sedimento en láminas portaobjetos y fueron cubiertas con cubre objetos. Posteriormente se evaluó bajo un microscopio.

3. En el microscopio se observó en forma de zigzag hasta la última parte recubierta. Finalmente se reportó como la presencia o ausencia según estructura.

4. Se anotó y evaluó.

Para el análisis estadístico se utilizó frecuencias absolutas y relativas, así como la prueba de Chi cuadrado y el coeficiente $V$ de Cramer.

Como consideraciones éticas, no se sometió a ningún riesgo a los adolescentes. Los padres fueron informados acerca del estudio y dieron su consentimiento voluntario antes de que los adolescentes se convirtieran en participantes de este estudio. La identidad de cada adolescente es anónima. 


\section{Resultados}

De los 92 adolescentes que participaron en este estudio, el $52 \%$ fue de género masculino $y$ el $48 \%$ de género femenino, y presentaron edades entre 13 a 17 años.

de los adolescentes con las concentraciones de arsénico en agua subterránea y no existe relación entre las concentraciones de hemoglobina y las concentraciones de arsénico en orina.

En la Tabla 1 se aprecia que no existe relación entre las concentraciones de hemoglobina

Tabla 1

Correlación de la hemoglobina con el arsénico en agua subterránea y orina

\begin{tabular}{lcccccccc}
\hline & \multicolumn{3}{c}{ Arsénico en agua subterránea } & \multicolumn{3}{c}{ Arsénico en orina } \\
\cline { 2 - 9 } Hemoglobina & \multicolumn{2}{c}{ Permisible } & \multicolumn{2}{c}{ Aceptable } & \multicolumn{2}{c}{ Aceptable } & \multicolumn{2}{c}{ No aceptable } \\
\cline { 2 - 9 } & $\%$ & Frec. & $\%$ & Frec. & $\%$ & Frec. & $\%$ & Frec. \\
\hline Normal & 71 & 12 & 86 & 6 & 54 & 7 & 100 & 11 \\
Leve & 18 & 3 & 0 & 0 & 23 & 3 & 0 & 0 \\
Moderado & 6 & 1 & 0 & 0 & 8 & 1 & 0 & 0 \\
Severo & 6 & 1 & 0 & 0 & 8 & 1 & 0 & 0 \\
Liq. elevado & 0 & 0 & 14 & 1 & 8 & 1 & 0 & 0 \\
\hline Total & 100 & 17 & 100 & 7 & 100 & 13 & 100 & 11 \\
\hline
\end{tabular}

Chi cuadrado $=4,639$

Chi cuadrado $=6,769$

Coeficiente V de Cramer $=0,440$

Coeficiente $V$ de Cramer $=0,531$

En la Tabla 2 se puede apreciar que no existe aluminio en agua subterránea y concentraciones relación entre las concentraciones de hemoglobina de aluminio en orina.

de los adolescentes con las concentraciones de

\section{Tabla 2}

Correlación de la hemoglobina con el aluminio en el agua subterránea y orina

\begin{tabular}{lcccccccc}
\hline & \multicolumn{3}{c}{ Aluminio en agua subterránea } & \multicolumn{3}{c}{ Aluminio en orina } \\
\cline { 2 - 9 } Hemoglobina & \multicolumn{2}{c}{ Permisible } & \multicolumn{2}{c}{ Aceptable } & \multicolumn{2}{c}{ Aceptable } & No aceptable \\
\cline { 2 - 9 } & $\%$ & Frec. & $\%$ & Frec. & $\%$ & Frec. & $\%$ & Frec. \\
\hline Normal & 69 & 11 & 88 & 7 & 74 & 17 & 100 & 1 \\
Leve & 19 & 3 & 0 & 0 & 13 & 3 & 0 & 0 \\
Moderado & 6 & 1 & 0 & 0 & 4 & 1 & 0 & 0 \\
Severo & 6 & 1 & 0 & 0 & 4 & 1 & 0 & 0 \\
Liq. elevado & 0 & 0 & 13 & 1 & 4 & 1 & 0 & 1 \\
\hline Total & 100 & 16 & 100 & 8 & 100 & 23 & 100 & 1 \\
\hline
\end{tabular}

Chi cuadrado $=4,750$

Chi cuadrado $=0,348$

Coeficiente $\mathrm{V}$ de Cramer $=0,445$

Coeficiente $\mathrm{C}$ de Cramer $=0,120$

Revista Médica Basadrina, 2021; 15(3):42-51. 
En la Tabla 3 se muestra la correlación entre la concentración de hemoglobina con la presencia o ausencia de Giardia lamblia y
Entamoeba histolytica por el consumo de agua subterránea y se encontró que no existe relación significativa.

Tabla 3

Correlación de la hemoglobina con la Giardia lamblia y Entamoeba histolytica en agua subterránea

\begin{tabular}{lcccccccc}
\hline & \multicolumn{3}{c}{ Giardia lamblia } & \multicolumn{2}{c}{ Entamoeba histolytica } \\
\cline { 2 - 9 } Hemoglobina & \multicolumn{2}{c}{ Ausencia } & \multicolumn{2}{c}{ Presencia } & \multicolumn{2}{c}{ Ausencia } & \multicolumn{2}{c}{ Presencia } \\
\cline { 2 - 9 } & $\%$ & Frec. & $\%$ & Frec. & $\%$ & Frec. & $\%$ & Frec. \\
\hline Normal & 73 & 16 & 9 & 2 & 73 & 16 & 100 & 2 \\
Leve & 14 & 3 & 0 & 0 & 14 & 3 & 0 & 0 \\
Moderado & 5 & 1 & 0 & 0 & 5 & 1 & 0 & 0 \\
Severo & 5 & 1 & 0 & 0 & 5 & 1 & 0 & 0 \\
Liq. elevado & 5 & 1 & 9 & 0 & 5 & 1 & 0 & 0 \\
\hline Total & 100 & 22 & 9 & 2 & 100 & 22 & 100 & 2 \\
\hline
\end{tabular}

Chi cuadrado $=0,727$

Coeficiente $V$ de Cramer $=0,174$

En las tablas 4 y 5 se muestra la correlación entre las concentraciones de hematocrito con las concentraciones permisibles o no permisibles de arsénico - aluminio en agua subterránea y
Chi cuadrado $=0,727$

Coeficiente $V$ de Cramer $=0,174$

\section{Tabla 4}

Correlación de la HCM con el arsénico en agua subterránea y orina

\begin{tabular}{|c|c|c|c|c|c|c|c|c|c|c|c|c|}
\hline \multirow{3}{*}{$\mathrm{HCM}$} & \multicolumn{4}{|c|}{ Arsénico en agua subterránea } & \multirow{2}{*}{\multicolumn{2}{|c|}{ Total }} & \multicolumn{4}{|c|}{ Arsénico en orina } & \multirow{2}{*}{\multicolumn{2}{|c|}{ Total }} \\
\hline & \multicolumn{2}{|c|}{ Permibible } & \multicolumn{2}{|c|}{ No permisible } & & & \multicolumn{2}{|c|}{ Aceptable } & \multicolumn{2}{|c|}{ No aceptable } & & \\
\hline & $\%$ & Frec. & $\%$ & Frec. & $\%$ & Frec. & $\%$ & Frec. & $\%$ & Frec. & $\%$ & Frec. \\
\hline Normal & 76 & 13 & 100 & 7 & 83 & 20 & 69 & 9 & 100 & 11 & 83 & 20 \\
\hline Bajo & 24 & 4 & 0 & 0 & 17 & 4 & 31 & 4 & 0 & 0 & 17 & 4 \\
\hline Total & 100 & 17 & 100 & 7 & 100 & 24 & 100 & 13 & 100 & 11 & 100 & 24 \\
\hline
\end{tabular}

\section{Tabla 5}

Correlación de la HCM con el aluminio en agua subterránea y orina

\begin{tabular}{|c|c|c|c|c|c|c|c|c|c|c|c|c|}
\hline \multirow{3}{*}{$\mathrm{HCM}$} & \multicolumn{4}{|c|}{ Aluminio en agua subterránea } & \multirow{2}{*}{\multicolumn{2}{|c|}{ Total }} & \multicolumn{4}{|c|}{ Aluminio en orina } & \multirow{2}{*}{\multicolumn{2}{|c|}{ Total }} \\
\hline & \multicolumn{2}{|c|}{ Permibible } & \multicolumn{2}{|c|}{ No permisible } & & & \multicolumn{2}{|c|}{ Aceptable } & \multicolumn{2}{|c|}{ No aceptable } & & \\
\hline & $\%$ & Frec. & $\%$ & Frec. & $\%$ & Frec. & $\%$ & Frec. & $\%$ & Frec. & $\%$ & Frec. \\
\hline Normal & 75 & 12 & 100 & 8 & 83 & 20 & 83 & 19 & 100 & 1 & 83 & 20 \\
\hline Bajo & 25 & 4 & 0 & 0 & 17 & 4 & 17 & 4 & 0 & 0 & 17 & 4 \\
\hline Total & 100 & 16 & 100 & 8 & 100 & 24 & 100 & 23 & 100 & 1 & 100 & 24 \\
\hline
\end{tabular}


En la Tabla 6 se muestra la correlación entre las concentraciones de hematocrito con la presencia o ausencia de Giardia lamblia y
Entamoeba histolytica en agua subterránea, y no existe la relación entre ambos.

\section{Tabla 6}

Correlación de la HCM con la Giardia lamblia y la Entamoeba histolytica en agua subterránea

\begin{tabular}{|c|c|c|c|c|c|c|c|c|c|c|c|c|}
\hline \multirow{3}{*}{$\mathrm{HCM}$} & \multicolumn{4}{|c|}{$\begin{array}{c}\text { Giardia lamblia en agua } \\
\text { subterránea }\end{array}$} & \multirow{2}{*}{\multicolumn{2}{|c|}{ Total }} & \multicolumn{4}{|c|}{$\begin{array}{c}\text { Entamoeba histolytica en agua } \\
\text { subterránea }\end{array}$} & \multirow{2}{*}{\multicolumn{2}{|c|}{ Total }} \\
\hline & \multicolumn{2}{|c|}{ Permibible } & \multicolumn{2}{|c|}{ No permisible } & & & \multicolumn{2}{|c|}{ Aceptable } & \multicolumn{2}{|c|}{ No aceptable } & & \\
\hline & $\%$ & Frec. & $\%$ & Frec. & $\%$ & Frec. & $\%$ & Frec. & $\%$ & Frec. & $\%$ & Frec. \\
\hline Normal & 82 & 18 & 100 & 2 & 83 & 20 & 83 & 19 & 100 & 1 & 83 & 20 \\
\hline Bajo & 18 & 4 & 0 & 0 & 17 & 4 & 17 & 4 & 0 & 0 & 17 & 4 \\
\hline Total & 100 & 22 & 100 & 2 & 100 & 24 & 100 & 23 & 100 & 1 & 100 & 24 \\
\hline
\end{tabular}

En las tablas 7 y 8 se muestran las correlaciones entre las concentraciones de $\mathrm{CHCM}$ con concentraciones permisibles y no permisibles de arsénico - aluminio en agua subterránea; concentraciones aceptables y no aceptables en la orina de los adolescentes, y en ningún caso se encontró relación.

\section{Tabla 7}

Correlación de la CHCM con el arsénico en agua subterránea y orina.

\begin{tabular}{|c|c|c|c|c|c|c|c|c|c|c|c|c|}
\hline \multirow{3}{*}{$\mathrm{CHCM}$} & \multicolumn{4}{|c|}{ Arsénico en agua subterránea } & \multirow{2}{*}{\multicolumn{2}{|c|}{ Total }} & \multicolumn{4}{|c|}{ Arsénico en orina } & \multirow{2}{*}{\multicolumn{2}{|c|}{ Total }} \\
\hline & \multicolumn{2}{|c|}{ Permibible } & \multicolumn{2}{|c|}{ No permisible } & & & \multicolumn{2}{|c|}{ Aceptable } & \multicolumn{2}{|c|}{ No aceptable } & & \\
\hline & $\%$ & Frec. & $\%$ & Frec. & $\%$ & Frec. & $\%$ & Frec. & $\%$ & Frec. & $\%$ & Frec. \\
\hline Normal & 71 & 12 & 100 & 7 & 79 & 19 & 62 & 8 & 100 & 11 & 79 & 19 \\
\hline Bajo & 29 & 5 & 0 & 0 & 21 & 5 & 38 & 5 & 0 & 0 & 21 & 5 \\
\hline Total & 100 & 17 & 100 & 7 & 100 & 24 & 100 & 13 & 100 & 1 & 100 & 24 \\
\hline
\end{tabular}

\section{Tabla 8}

Correlación de la CHCM con el aluminio en agua subterránea y orina

\begin{tabular}{|c|c|c|c|c|c|c|c|c|c|c|c|c|}
\hline \multirow{3}{*}{$\mathrm{CHCM}$} & \multicolumn{4}{|c|}{ Aluminio en agua subterránea } & \multirow{2}{*}{\multicolumn{2}{|c|}{ Total }} & \multicolumn{4}{|c|}{ Aluminio en orina } & \multirow{2}{*}{\multicolumn{2}{|c|}{ Total }} \\
\hline & \multicolumn{2}{|c|}{ Permibible } & \multicolumn{2}{|c|}{ No permisible } & & & \multicolumn{2}{|c|}{ Aceptable } & \multicolumn{2}{|c|}{ No aceptable } & & \\
\hline & $\%$ & Frec. & $\%$ & Frec. & $\%$ & Frec. & $\%$ & Frec. & $\%$ & Frec. & $\%$ & Frec. \\
\hline Normal & 69 & 11 & 100 & 8 & 79 & 19 & 78 & 18 & 100 & 1 & 79 & 19 \\
\hline Bajo & 31 & 5 & 0 & 0 & 21 & 5 & 22 & 5 & 0 & 0 & 21 & 5 \\
\hline Total & 100 & 16 & 100 & 8 & 100 & 24 & 100 & 23 & 100 & 1 & 100 & 24 \\
\hline
\end{tabular}

En las tablas 9 se muestran las correlaciones entre las concentraciones de $\mathrm{CHCM}$ con la ausencia y presencia de Giardia lamblia y
Entamoeba histolytica en agua subterránea que consumen los adolescentes, y en ningún caso se encontró relación. 
Tabla 9

Correlación de la CHCM con la Giardia lamblia y la Entamoeba histolytica en agua subterránea

\begin{tabular}{|c|c|c|c|c|c|c|c|c|c|c|c|c|}
\hline \multirow{3}{*}{$\mathrm{HCM}$} & \multicolumn{4}{|c|}{$\begin{array}{c}\text { Giardia lamblia en agua } \\
\text { subterránea } \\
\end{array}$} & \multicolumn{6}{|c|}{$\begin{array}{l}\text { Entamoeba histolytica en agua } \\
\text { subterránea }\end{array}$} & \multirow{2}{*}{\multicolumn{2}{|c|}{ Total }} \\
\hline & \multicolumn{2}{|c|}{ Ausencia } & \multicolumn{2}{|c|}{ Presencia } & \multirow[b]{2}{*}{$\%$} & \multirow[b]{2}{*}{ Frec. } & \multicolumn{2}{|c|}{ Ausencia } & \multicolumn{2}{|c|}{ Presencia } & & \\
\hline & $\%$ & Frec. & $\%$ & Frec. & & & $\%$ & Frec. & $\%$ & Frec. & $\%$ & Frec. \\
\hline Normal & 77 & 17 & 100 & 2 & 79 & 19 & 78 & 18 & 100 & 1 & 79 & 19 \\
\hline Bajo & 23 & 5 & 0 & 0 & 21 & 5 & 22 & 5 & 0 & 0 & 21 & 5 \\
\hline Total & 100 & 22 & 100 & 2 & 100 & 24 & 100 & 23 & 100 & 1 & 100 & 24 \\
\hline
\end{tabular}

\section{Discusión}

En la investigación de A. Quiroga y E. Leonarduzzi, en una población expuesta a arsénico por consumo de agua subterránea no detectaron cambios a nivel hematológico como la hemoglobina, hematocrito y las constantes corpusculares. $^{9}$ La investigación presente determina un resultado similar porque encontramos concentraciones normales de hemoglobina, hematocrito y constantes corpusculares en los adolescentes que consumen agua subterránea.

En presencia de arsénico inorgánico y al consumo los parámetros como la hemoglobina se encuentran disminuidos, en los resultados se muestra que se encontro la hemoglobina en mayor proporción como normal con relación a las concentraciones no permisibles de arsénico en agua subterránea. Así, se consideró las constantes corpusculares como el volumen corpuscular medio, hemoglobina corpuscular medio y la concentración de la hemoglobina corpuscular medio, que se encontro dentro de los valores referenciales considerados como normales con relación a las concentraciones no permisibles de arsénico en agua subterránea. Sin embargo, se encontró un porcentaje menor de hemoglobina moderada, hemoglobina leve y hemoglobina severa con relación al arsénico en agua subterránea permisible.

La Dirección General de Salud Ambiental (DIGESA), advierte el consumo de arsénico en agua subterránea, por lo que se encontró la hemoglobina normal en mayor proporción con relación a las concentraciones permisibles para arsénico en orina. ${ }^{10}$ Además, se encontró la hemoglobina normal en mayor proporción con relación a las concentraciones no aceptables para arsénico en orina. Benito, en su investigación tuvo como objetivo disminuir el arsénico en las aguas subterráneas de Juliaca-Taparachi, cuyas aguas tienen concentraciones de arsénico de $0,06 \mathrm{mg} / \mathrm{L}$ la cual están por encima de los límites máximos permisibles de acuerdo a la Norma Técnica Peruana. ${ }^{11}$ En la presente investigación se demuestra que las concentraciones de arsénico en agua subterránea se encuentran en valores permisibles en mayor proporción, es decir, en concentraciones menores a $0,01 \mathrm{mg} / \mathrm{L}$ que son permisibles de acuerdo a la Organización Mundial de la Salud. ${ }^{12}$

Asimismo, en la investigación de D. Arnao determino que es necesario el conocimiento de la calidad del agua para el consumo humano, para mantener las condiciones de salud adecuadas, practicarlas y difundirlas en la urbanización Taparachi III Juliaca. ${ }^{13} \mathrm{En}$ la presente investigación se demuestra el conocimiento de una hemoglobina normal en mayor proporción con relación a la presencia y la ausencia de Giardia lamblia y Entamoeba histolytica en el agua subterránea de la Urbanización Taparachi III Juliaca.

DIGESA Juliaca determino los niveles no permisibles de aluminio en agua subterránea en la urbanización Taparachi III. ${ }^{14}$ En la presente investigación la hemoglobina se encontró normal en mayor proporción con relación a las concentraciones permisibles para aluminio en agua subterránea, asimismo, se encontró la hemoglobina normal en mayor proporción para las concentraciones no permisibles en agua subterránea. La investigación encontro la hemoglobina normal en mayor proporción con relación a las concentraciones aceptables y no aceptables de aluminio en orina, y el aluminio en orina se encontró como mayor proporción como aceptable. 


\section{Conclusiones}

Se concluye que no existe relación entre la anemia ferropénica y el consumo de agua subterránea en los adolescentes de la urbanización Taparachi III Juliaca, Puno, en el 2019. Los adolescentes se encuentran con hemoglobina y constantes corpusculares dentro de los valores de referencia considerados como normales. La hemoglobina encontrada como leve, moderada y severa son por cualquier otra causa.

Existe concentraciones permisibles de arsénico en agua subterránea. Las concentraciones encontradas no aceptables de arsénico en orina en los adolescentes son por el arsénico de tipo orgánico o los adolescentes están siendo tolerantes al arsénico inorgánico que se encuentran en concentraciones muy bajas siendo permisibles en agua subterránea. Las concentraciones de aluminio se encuentran permisibles. El aluminio encontrado en concentraciones no permisibles en agua subterránea es por cualquier otra causa externa. Las concentraciones de aluminio son aceptables en orina de los adolescentes.

Se encontró ausencia de Giardia lamblia y Entamoeba histolytica en agua subterránea de la Urbanización Taparachi III.

\section{Referencias}

1. Nordberg G, Langard S, Sunderman FW, Mager Stellman J, Osinsky D, Markkanen $P$, et al. Metales: propiedades quimicas y toxicidad. Encicl salud y Segur en el Trab [Internet]. 2001;1-76. Available from: https://www.insst. es/documents/94886/162520/Capítulo+63.+Metales+propiedades+químicas+y+toxicidad

2. Failace R. Hemograma. Manual de interpretación. 2011;1-463.

3. Fallis A. Distribución del arsénico en las regiones ibérica e iberoamericana. J Chem Inf Model [Internet]. 2013;53(9):1689-99. Available from: https://www.researchgate.net/publication/258702251_Distribucion_del_arsenico_ en_las_regiones_lberica_e_lberoamericana

4. Viva Q, Nesse A, Garbossa G, Pérez G, Vittori D. Aluminio: ¿culpable o inocente? Química Viva [Internet]. 2003;2(1):9-16. Available from: https://www.researchgate.net/publica-
tion/26616461_Aluminio_culpable_o_inocente

5. Agudelo GM, Cardona OL, Posada M, Montoya MN, Ocampo NE, Marín CM, et al. Prevalencia de anemia ferropénica en escolares y adolescentes, Medellín, Colombia, 1999. Rev Panam Salud Publica/Pan Am J Public Heal [Internet]. 2003;13(6):376-86. Available from: https://iris. paho.org/handle/10665.2/8428

6. Botero D, Respreto M. Parasitosis humanas incluye animales venenosos y ponzoñosos [Internet]. 1386.32 ، 117.

7. Organización Mundial de la Salud. Guias de la OMS para la calidad del agua potable. Agua Saneam y salud ASS [Internet]. 2012;243-363. Available from: http://www.who.int/water_sanitation_health/dwq/guidelines/es/index.html

8. Indice Biologico de Exposicion | Biotransformación | Ciencias de la tierra y de la vida [Internet]. [cited 2020 Oct 18]. Available from: https://es.scribd.com/document/297384258/lndice-Biologico-de-Exposicion

9. Quiroga A, Leonarduzzi E, ... IL-R de S, 2020 undefined. Evaluación de poblaciones rurales expuestas a arsénico en el agua de consumo en la Provincia de Santa Fe, Argentina. Estrategias de comunicación y prevención. ojs.diffundit.com [Internet]. [cited 2021 Jul 27]; Available from: https://ojs.diffundit.com/index.php/rsa/article/view/1038

10. DIGESA. DIGESA continúa vigilando la calidad del agua para consumo humano a fin de garantizar la salud del país | DIGESA [Internet]. 2020 [cited 2020 Oct 18]. Available from: http://www. digesa.minsa.gob.pe/noticias/Octubre2020/ nota62.asp

11. BENITO. Facultad de Ingeniería Facultad de Ingeniería. Ucv [Internet]. 2014;0-116. Available from: http://repositorio.ucv.edu.pe/handle/20.500.12692/32871

12. OMS. Arsénico [Internet]. 2018 [cited 2020 Oct 18]. Available from: https://www.who.int/es/ news-room/fact-sheets/detail/arsenic 
13. Calidad Del Agua Para Consumo Humano. Rev Científica Investig Andin [Internet]. 2019;18(1):1-11. Available from: https://revistas.uancv.edu.pe/index.php/RCIA/article/ view/602/529
14. Por redacción Web. Agua de Juliaca tiene demasiado aluminio y arsénico perjudiciales para la salud [Internet]. 2017 [cited 2020 Oct 18]. Available from: https://www.diariosinfronteras. pe/2017/11/25/agua-de-juliaca-tiene-demasiado-aluminio-y-arsenico-perjudiciales-para-la-salud/

\section{Correspondencia:}

yesirossy@hotmail.com
Fecha de recepción: 31/03/2021

Fecha de aceptación: 30/07/2021 